\title{
Determinación del índice cardíaco vertebral en perros de raza mestiza
}

\author{
Vertebral cardiac index determination in mongrel dogs

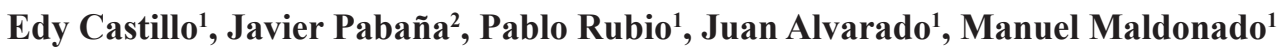 \\ RESUMEN
}

El objetivo de esta investigación fue determinar el índice cardíaco vertebral (ICV) en perros de raza mestiza, en la ciudad de Cuenca a una altitud de $2560 \mathrm{msnm}$ y comparar el resultado con diferentes estudios realizados en investigaciones anteriores a diferentes altitudes. El tamaño de la de la silueta cardiaca fue evaluado por el método de Buchanan y Bucheler (1995), que relacionan el alto y ancho del corazón con el número de vértebras torácicas en perros Beagle. Para este estudio se empleó una investigación descriptiva de corte transversal, seleccionándose treinta y tres perros de raza mestiza clínicamente sanos, sin discriminación de sexo, y con edades comprendidas entre uno a cinco años. Los animales fueron sometidos a un examen clínico y radiográfico del tórax con una proyección lateral derecha. Los valores del índice cardíaco vertebral obtenidos presentaron una distribución normal, con promedio de 9,38 vértebras y una desviación estándar de $\pm 0,15$. El valor mínimo encontrado fue igual a 8,8 vértebras y el máximo fue igual a 9,8 vértebras. Se realizó el análisis de confianza de los datos del ICV de acuerdo al peso, edad y sexo, comprobando que la medida obtenida como rango general del ICV es estandarizada en perros de raza mestiza y no está influenciada por los mismos.

PALABRAS CLAVE: Tórax, altitud, radiografía, silueta cardiaca.

\section{SUMMARY}

The investigation objective was to determine the vertebral cardiac index (VCI) in mongrel dogs, from Cuenca city at 2560 meters above sea level and compare it with the results of different studies carried out with different conditions and altitudes. The heart's size was evaluated by Buchanan and Bucheler method (1995), that relate the height and width of the heart, with a specific number and position of thoracic vertebrae in Beagle dogs. For this descriptive study there were selected thirty-three clinically healthy mongrel dogs, without discrimination of sex, and an age range from one to five years. The animals were clinical tested and after that a radiographic examination of the thorax with a right lateral projection was made. The vertebral cardiac index values obtained, showed a normal distribution, with an average of 9.38 vertebrae and a standard deviation of \pm 0.7 . The minimum value found was equal to 8.8 vertebrae and the maximum was equal to 9.8 vertebrae. The confidence analysis of the VCI verifies that the general range VCI measure is not influenced by weight, age or sex; and can be standardized in mongrel dogs that live in this conditions.

KEY WORDS: thorax, meters above sea level, radiographic, hearts size.

Carrera de Medicina Veterinaria, Unidad Académica de Ciencias Agropecuarias, Universidad Católica de

Cuenca. Cuenca, Ecuador.

2 Veterinaria Crespo. Cuenca, Ecuador. 


\section{INTRODUCCIÓN}

Las radiografías torácicas son esenciales en la evaluación de los animales en los que se sospecha de una enfermedad cardíaca (Carrillo, Grandez \& Dávila,2011). Sin embargo, en ocasiones los estudios pueden ser frustrantes porque los cambios radiológicos pueden no ser específicos (Escobedo, 2009). Este tipo de instrumento diagnóstico nos ayuda a valorar la dimensión y la forma de la silueta del corazón, sus estructuras adyacentes como los pulmones y diferentes tipos de vasos sanguíneos. De igual forma nos permite valorar la gravedad de una cardiopatía, la evaluación y eficacia del tratamiento propuesto (García, 2013).

Para la evaluación radiográfica cardíaca, se emplea las proyecciones latero lateral derecha (L-L) y dorso ventral (D-V), pudiendo medirse el índice cardíacovertebral (ICV) con la primera proyección (Carrillo et al., 2011). La misma que debe realizarse en fase inspiratoria, debiendo considerarse que una toma en expiración da una falsa percepción de cardiomegalia, debido a que la cavidad torácica disminuye al no existir una cantidad considerable de aire, sin embargo, el tamaño cardíaco sigue siendo el mismo. Radiológicamente el corazón es el órgano mediastínico más grande de la cavidad torácica, posee una forma cónica y se encuentra ubicado en forma oblicua, su base está localizada dorso-craneal y caudo-ventral en relación al ápice. La silueta cardíaca incluye el pericardio que contiene en su interior líquido y grasa (Agut y Soler, 2014a,b).

La evaluación radiográfica del tórax es en una técnica diagnóstica idónea, que requiere de buen conocimiento anatómico debido a las particularidades de cada raza. La calidad de imagen radiológica depende de varios factores como la técnica, posicionamiento, el has de rayos $\mathrm{X}$ centrado, un potencial alto de $\mathrm{mA}$ y Kv razonable acorde al ancho del tórax, condición corporal y masa muscular del paciente, con un bajo mAs (Carrera, 2014).

En la radiografía torácica se debe observar perfectamente las líneas del diafragma sin estar expuesto ningún órgano o tejido. Las costillas no deben presentar ninguna imagen borrosa y los lóbulos craneales deben tener un tono marcado de nitidez. Las uniones costocondrales de cada lado deben estar al mismo nivel, y la columna no debe estar opacada por la curvatura dorsal de las costillas. En la radiografía de tórax, se debe verificar que no existan variaciones anatómicas para lo cual se deben tomar en cuenta los siguientes parámetros, edad, sexo, raza, conformación anatómica y condición corporal (Exposito, 2017), y se recomienda usar un elevado kilo-voltaje de 90 a 150 y bajo mili amperaje.

Para la valoración de la silueta cardíaca existen métodos subjetivos como: 1) número de espacios intercostales, en el cual se valora el tamaño del corazón en función de los espacios intercostales que ocupa su silueta, siendo el diámetro cráneo-caudal del corazón de aproximadamente 2.5 espacios intercostales en razas de perros de tórax estrechos y hasta 3.5 en razas de perros de tórax ancho. 2) Contacto esternal, se valora el tamaño del corazón en función del número de esternebras que están en contacto con la silueta cardíaca. Se considera cardiomegalia cuando el corazón contacta con más de tres esternebras. 3) Ratiocorazón-tórax, se compara el tamaño del corazón con el tamaño de la cavidad torácica, para ello se traza una línea que una la carina y el ápex cardiaco (S1) y otra línea al mismo nivel que una los límites ventral y dorsal de la cavidad torácica (S2), el cociente S1/ S2 proporciona la ratio. Se describe cardiomegalia cuando la silueta cardiaca es superior al 75\% del tórax. La subjetividad de los criterios radiográficos para valorar la cardiomegalia generó la necesidad de disponer de algún criterio radiográfico objetivo que pudiera precisar mejor la presencia o ausencia de cardiomegalia eliminando factores de variación (raza, sexo, peso edad). En este sentido, Buchanan y Bucheler (1995) determinaron el índice cardiaco vertebral (ICV) como nuevo método para evaluar el tamaño cardíaco de forma más objetiva. Establecieron el eje cardiaco mayor partiendo del límite ventral de la carina hasta el punto más distante del ápex cardiaco $y$, perpendicular a este, el eje cardiaco menor en la zona de máxima amplitud cardiaca. Ambos ejes se traspusieron de forma paralela a la columna vertebral, al inicio del cuerpo de la cuarta vértebra torácica (T4) y se cuantificó el número de cuerpos vertebrales que ocupa la suma de ambos ejes, obteniendo un rango de normalidad de $9.7 \pm 0.5$ vertebras (v), con un rango de distribución normal entre 8.5 y 10.6 cuerpos vertebrales (Liste, 2010).

\section{MATERIAL Y MÉTODOS}

La investigación trabajó con 33 perros de ambos sexos, de raza mestiza, clínicamente sanos, con pesos de 6 a $15 \mathrm{~kg}$ y edades de 1 a 5 años. Se seleccionaron pacientes sin antecedentes de patologías cardiacas, 
a los cuales se realizaron análisis físicos, clínicos, y perfil hematológico para comprobar su estado de salud.

Las radiografías fueron tomadas con una proyección latero-lateral derecha (LL-D), colocando al paciente en decúbito lateral derecho y posicionando el haz de rayos X en la parte caudal de la escapula, la colimación incluyó cranealmente la entrada torácica y caudalmente el diafragma en su conjunto. Ventralmente se incluyó el esternón y dorsalmente la columna vertebral, utilizando para el efecto un equipo Vetter Rems, de fabricación argentina, modelo 2005, el cual tiene una capacidad de mili amperaje de 20 y un kilo voltaje de 95 . Las radiografías fueron evaluadas cuantitativamente, por el método de ICV descrito por Buchanan y Bucheler (1995), tomando en cuenta las medidas del eje largo y el eje corto, el ICV determinado se calculó midiendo el número de vertebras torácicas que abarcan estas dos medidas, colocándose al inicio del cuerpo de la cuarta vértebra torácica (T4) y obteniéndose un promedio de $9.38 \pm 0.7$ vertebras.

\section{RESULTADOS}

Los rangos, mínimo, máximo, numero, promedio y desviación estándar del peso, eje mayor, eje menor, numero de vertebras menor, numero de vertebras mayor y ICV se expresan en la tabla 1.

Los análisis de varianza se realizaron en forma conjunta sin presentar diferencias significativas $(\mathrm{P}>0.05)$ entre cada una de las variables. Al combinar

Tabla1. Estadística descriptiva de las variables de estudio

\begin{tabular}{lccc}
\hline & Mínimo & Máximo & Promedio \pm DS \\
\hline Peso $(\mathrm{kg})$ & 6 & 15 & $10.9 \pm 0.25$ \\
Eje mayor $(\mathrm{cm})$ & 66 & 103.5 & $83.0 \pm 0.35$ \\
Vertebras mayor & 4.8 & 5.4 & $5.1 \pm 0.45$ \\
Eje menor $(\mathrm{cm})$ & 50.9 & 85.6 & $67.6 \pm 0.55$ \\
Vertebras menor & 3.9 & 4.6 & $4.2 \pm 0.65$ \\
ICV & 8.8 & 9.8 & $9.4 \pm 0.15$ \\
\hline
\end{tabular}

Tabla 2. Intervalos de confianza del índice cardíaco vertebral (ICV) de acuerdo al sexo e interacción sexo-edad y sexo-peso.

\begin{tabular}{lrcc}
\hline & ICV & Inferior & Superior \\
\hline Hembras & 9.5 & 9.4 & 9.6 \\
Machos & 9.3 & 9.2 & 9.4 \\
Hembras 15 a 24 meses & 9.5 & 9.1 & 9.9 \\
Hembras > 24 meses & 9.5 & 9.3 & 9.6 \\
Machos de 15 a 24 meses & 9.3 & 9.1 & 9.4 \\
Hembra 6-10kg & 9.5 & 9.3 & 9.7 \\
Hembra > 10 kg & 9.4 & 9.2 & 9.5 \\
Macho 6-10kg & 9.2 & 9.1 & 9.4 \\
Macho > 10 kg & 9.4 & 9.2 & 9.5 \\
\hline
\end{tabular}

Tabla 3. Correlaciones significativas $(\mathrm{p}<0.01)$ entre dimensiones.

\begin{tabular}{lll}
\hline Variables & Eje Mayor & Eje menor \\
\hline Peso & 0,617 & 0,584 \\
Eje Mayor & 1 & 0,895 \\
Eje menor & 0,895 & 1 \\
\hline & Vertebras Mayores & Vertebras Menores \\
\hline Dimensión Final ICV & 0,588 & 0,803 \\
\hline
\end{tabular}


Tabla 4. Promedios de diferentes estudios comparados con el ICV de perros de raza mestiza

\begin{tabular}{lcccc}
\hline & Promedio & Inferior & Superior & Valor P \\
\hline ICV perros raza mestiza & 9.38 & 9.28 & 9.48 & $<0.01$ \\
Labrador (Bodh et al., 2016) & 10.39 & 10.20 & 10.58 & $<0.01$ \\
Varias razas (Buchanan y Bucheler, 1995) & 9.70 & 9.20 & 10.20 & $<0.01$ \\
Pitbull (Lahm et al.,2011) & 10.90 & 10.50 & 11.30 & $>0.05$ \\
\hline
\end{tabular}

los factores se determinó que la interrelación entre las variables tampoco influye significativamente ( $\mathrm{P}>0.05)$, como se expresa en la tabla 2, que relaciona el ICV por sexos y la interacción sexo-edad y sexopeso.

El peso guarda una correlación positiva y alta $(\mathrm{P}<0.01)$ con respecto al eje mayor $(0.617)$ y eje menor (0.584), mientras la relación de los ejes entre si es la más alta de todas $(0.895)$. La dimensión de las vértebras mayores $(0.588)$ y menores $(0.803)$ por su parte guardan una correlación positiva $(\mathrm{P}<0.01)$ con respecto a la dimensión final del ICV, tal como se encuentra en la tabla 3.

Los resultados del índice cardiaco vertebral propuestos en esta investigación tienen un promedio de $9.38 \pm 0.1$ cuerpos vertebrales. Estos valores son inferiores a los expuestos en la tabla 4, donde se observan los valores promedios, mínimos y máximos de otros estudios similares.

\section{DISCUSIÓN}

Buchanan y Bucheler (1995), definieron en su estudio en perros Beagle una media general de $9.7 \pm$ 0.5 ; posteriormente en el Instituto de Investigación Veterinaria de la India (Bodh et al., 2016), indicaron que existe diferencias del ICV en cada raza. Este mismo estudio incluyó perros de raza mestiza; obteniendo una medida de $9.82 \pm 0.21$. A pesar de que estos resultados son superiores a los obtenidos en esta investigación $(9.38 \pm 0.1)$, no guardan una diferencia estadística significativa entre los mismos.

Los valores obtenidos en la presente investigación se pueden correlacionar con lo investigado por Bodh et al., (2016), quienes indican que la especificidad de las razas influye en el ICV, tal como lo comprueba Fonseca-Pinto (2004), quien halló un ICV en perros raza Poodle de $10.12 \pm 0.5$; en tanto que Guzmán y Rodríguez (2011), lo determinó en 8.7. Azevedo et al., (2016), compararon estos valores en la raza Poodle donde obtuvieron un ICV de $9.7 \pm 0.7$. Castro, Torres,
Araújo, Muzzi \& Silva (2011), determinaron un valor medio de $9.9 \pm 0.6$ para la raza Yorkshire Terrier. Las diferencias de estos valores se justifican en las características específicas de cada raza ya que estudios como el de Lahm, Caludino \& Melussi (2011), determinaron el ICV para perros de raza Pitbull en 10.9 $\pm 0.4 \mathrm{y}$, el de Bodh et al., (2016), determinó un valor de $10.39 \pm 0.2$ para perros Labradores; sin embargo, no existen diferencias en cuanto a peso, edad y sexo.

Consecuentemente a pesar de la variabilidad fenotípica de los animales de raza mestiza, no existe diferencia entre sí, coincidiendo con lo afirmado por Bodh et al., (2016), quienes indican que la determinación del índice cardíaco vertebral en perros de raza mestiza, no se ve influenciada por el peso, edad, sexo, al igual que la altitud.

Se recomienda realizar nuevos estudios en un amuestra mayor de animales donde se evalué el tipo de tórax de los pacientes.

Agradecimientos: Dejamos constancia de nuestro agradecimiento a la Clínica Veterinaria de la Universidad Católica de Cuenca y a la Clínica Veterinaria Austrovet, por el apoyo brindado para el desarrollo de la presente investigación, permitiendo el uso de sus instalaciones.

\section{Correspondencia:}

Edy Castillo

Correo electrónico: ecastilloh@ucacue.edu.ec

\section{REFERENCIAS BIBLIOGRAFICAS}

1. Agut, A. \& Soler, M. (2014a). Diagnóstico por imagen en pequeños animales. Producción de los rayos X: obtención de la imagen radiológica. (pp.449458). España: Multimédica Ediciones Veterinarias,

2. Agut, A. \& Soler, M. (2014b). Diagnóstico por imagen en pequeños animales. Radiología cardíaca. España: Multimédica Ediciones Veterinarias.

3. Azevedo, G. M., Pessoa, G. T., da Silva Moura, L., 
Sousa, F. D. C. A., de Sá Rodrigues, R. P., Sanches, M. P., ... \& Alves, F. R. (2016). Comparative study of the Vertebral Heart Scale (VHS) and the Cardiothoracic Ratio (CTR) in healthy poodle breed dogs. Acta Scientiae Veterinariae, 44(1), 7.

4. Bodh, D., Chaudhary, J., Bist, D., Bashir, M., Chandra, A., \& Hoque, M. (2016). Vertebral scale system to measure heart size in thoracic radiographs of Indian Spitz, Labrador retriever and Mongrel dogs. Veterinary World, 9, 371-376.

5. Buchanan, J. \& Bücheler, J. (1995). Vertebral scale system to measure canine heart size in radiographs. Journal of the American Veterinary Medical Association, 206, 194-199.

6. Carrera, I. (2014). Interpretacion radiologíca de la cavidad torácica y otras modalidades de imagen torácica en: Diagnóstico por imagen en pequeños animales. (pp. 449-458). España: Multimédica Ediciones Veterinarias,.

7. Carrillo, L., Grandez, R., \& Dávila R. (2011). Parámetros electrocardiográficos y radiográficos cardíacos en la raza perro sin pelo del Perú. Rev Inv Vet Perú, 22, 89-96.

8. Castro M., Torres R., Araújo R., Muzzi R., \& Silva E. (2011). Avaliação radiográfica da silhueta cardíaca pelo método vertebral heart size em cães da raça Yorkshire Terrier clinicamente normais. Arq Bras Med Vet Zootec, 63, 850-857.
9. Escobedo, G. (2009). Estudio electrocardiográfico, radiográfico y ecocardiográfico en perros geriátricos de raza pequeña con sintomatología de bulbopatía mitral crónica adquirida. Tesis para obtener el grado de Maestro en Ciencias Pecuarias por la Universidad Autónoma de Aguas Calientes.

10. Exposito, J. (2017). Limitaciones de la radiología torácica para determinar la causa de la cardiomegalia. Clincardiovet, 8, 2-10.

11. Fonseca, A. (2004). Avaliação radiográfica da silhueta cardíaca pelo método de mensuração VHS (vertebral heart size) em cães da raça Poodle clinicamente normáis. Brazilian Journal of Veterinary Research and Animal Science, 41, 261-267.

12. García, I. (2013). Atlas de interpretación radiológica en pequeños animales. España: Servet.

13. Guzman, K., \& Rodriguez, A. (2011). Determinación del Indice Vertebral Cardiaco y su variabilidad interobservacional en 30 caninos de raza French Poodle sanos y con enfermedad de la válvula mitral. Bogotá, Colombia: Universidad de la Salle.

14. Lahm, M., Caludino, J., \& Melussi, M. (2011). Mensuração do tamanho cardíaco pelo método VHS (vertebral heart size) em cães sadios da raça American pit bull terrier. Ciência Rural, 41, 127-131.

15. Liste, F. (2010). Atlas veterinario de diagnóstico por imagen. Zaragoza: Servet. 